Copyright $(2005$ IEEE. Reprinted from Proceedings of the 29th IEEE International Conference on Acoustics, Speech, and Signal Processing, Vol. 4, pp. 25-28, Montreal, Canada, May 2004.

This material is posted here with permission of the IEEE. Such permission of the IEEE does not in any way imply IEEE endorsement of your university's web sites products or services. Internal or personal use of this material is permitted. However, permission to reprint/republish this material for advertising or promotional purposes or for creating new collective works for resale or redistribution must be obtained from the IEEE by writing to pubs-permissions@ieee.org.

By choosing to view this document, you agree to all provisions of the copyright laws protecting it. 


\title{
BOUNDARY CONDITIONS IN A MULTI-DIMENSIONAL DIGITAL WAVEGUIDE MESH
}

\author{
Antti Kelloniemi ${ }^{1)}$, Damian T. Murphy ${ }^{2)}$, Lauri Savioja ${ }^{1)}$, Vesa Välimäki ${ }^{3}$ ) \\ 1) Helsinki University of Technology, Telecommunications Software and Multimedia Lab. \\ P.O.Box 5400, FIN-02015 HUT, Finland \\ 2) University of York, Media Engineering Research Group, Dept. of Electronics \\ Heslington, York, YO10 5DD, UK \\ ${ }^{3}$ Helsinki University of Technology, Lab. of Acoustics and Audio Signal Processing \\ P.O. Box 3000, FIN-02015 HUT, Finland
}

\begin{abstract}
The digital waveguide mesh is a modeling technique suitable for simulation of wave propagation in an acoustic system. In this work artificial boundary conditions are constructed for the digital waveguide mesh. Absorbing boundary conditions are evaluated and a new method for adjusting the reflection coefficient at values $0 \leq r \leq 1$ is introduced. The frequency dependent error level of this method was minimized with use of a second-order FIR filter.
\end{abstract}

\section{INTRODUCTION}

The digital waveguide mesh is a wave-based acoustic modeling technique [1,2] that, when compared to geometric acoustic methods such as ray-tracing and the image source method, has the benefit of incorporating diffraction and interference effects $[2,3]$ as a natural consequence of the model's emerging behaviour. The method has been extended to two and three dimensions from the digital waveguide method [4] used for simulation of one-dimensional musical instruments, such as plucked string instruments and woodwinds.

The original rectangular mesh suffers from direction dependent dispersion. This can be reduced by using triangular or interpolated mesh structures and frequency warping techniques [5]. These methods enhance the behavior of the mesh as a model of wave propagation in a homogenous medium. The same dispersion also affects the reflections at the boundaries.

Currently there are only few studies of boundary conditions in the mesh. In this work approximation methods of absorbing and reflecting boundary conditions are evaluated. Absorbing boundaries are needed for mesh truncation, when calculations of infinite space are made using finite mesh sizes. Different reflection characteristics are of

\footnotetext{
The work was funded by the Academy of Finland (no. 201050).
}

use in simulations where obstacles, such as walls and furniture in room acoustics, are involved. Earlier methods for simulating such boundaries gave rise to large magnitude errors. In this paper a new method which diminishes the error dramatically is introduced and evaluated.

This paper is organized as follows. Section 2 describes the interpolated rectangular mesh structure. The novel method for arbitrary boundary conditions is presented in Section 3. The new technique is validated by simulations discussed in Section 4. Section 5 concludes the article.

\section{INTERPOLATED MULTI-DIMENSIONAL WAVEGUIDE MESH}

A multi-dimensional rectangular digital waveguide mesh is a regular array of 1-D digital waveguides arranged along each perpendicular dimension, interconnected with unit delay elements. The difference equation for the nodes of an $\mathrm{N}$-dimensional rectangular mesh is given by:

$$
p_{c}(n)=\frac{1}{N} \sum_{l=1}^{2 N} p_{l}(n-1)-p_{c}(n-2),
$$

where $p$ represents the signal pressure at a junction at time step $n$, subscript $c$ denotes the junction to be calculated and index $l$ denotes its $2 N$ axial neighbours [2].

In the interpolated mesh there are also connections from a node to all its diagonal neighbours [5]. This way the direction dependent dispersion error created in the rectangular mesh is effectively diminished. The difference equation for the nodes of a two dimensional interpolated rectangular mesh is given by:

$$
p_{c}(n)=\frac{2}{H} \sum_{l=1}^{3} \sum_{k=1}^{3} h_{l, k} p_{l, k}(n-1)-p_{c}(n-2),
$$

where $h_{l, k}$ are weighting coefficients for each node [5] and $H=8$ is the number of wave propagation directions. 


\section{BOUNDARY CONDITIONS IN THE INTERPOLATED DIGITAL WAVEGUIDE MESH}

The first step towards frequency and angle dependent reflection conditions is to develop a technique for an arbitrary reflection coefficient. In this work we construct a new method based on absorbing boundary condition presented earlier by Murphy and Mullen [6].

The basic absorbing boundary condition (denoted as zero-order $\mathrm{ABC}$ ) has been derived from the discrete border condition of the acoustical pressure,

$$
p_{B}(n)=(1+r) p_{1}(n-1)-r p_{B}(n-2),
$$

where subscript $B$ denotes the border node, subscript 1 represents its perpendicular neighbour and $r$ is the reflection coefficient [2]. The two terms on the right side of Eq. (3) represent the sound pressure in front of the boundary one time step ago and the sound pressure reflected from the boundary two time steps ago respectively. Hence, with $r=0$ the last term is absent and we get the absorbing condition

$$
p_{B, a b s}(n)=p_{1}(n-1) .
$$

In simulations it has been noticed that the zero-order condition in Eq. (4) gives rise to reflections [2] and better absorption is needed for creating an adequate non-reflecting border. One possible solution is the one-way approximation of the wave equation obtained by calculating the border node value $p_{B, a b s}(n)$ as a Taylor series approximation of the past value of the adjacent node $p_{1}(n-1)$ [6]:

$$
\begin{gathered}
p_{B, a b s}(n)=p_{1}(n-1)+\Delta n p_{1}^{\prime}(n-1)+ \\
\frac{\Delta n^{2}}{2 !} p_{1}^{\prime \prime}(n-1)+\frac{\Delta n^{3}}{3 !} p_{1}^{\prime \prime \prime}(n-1)+\cdots
\end{gathered}
$$

For example, assuming that $\Delta n=1$, the first order solution of the series can be written as difference equation:

$$
\begin{aligned}
p_{B, a b s}(n)_{1} & =p_{1}(n-1)+p_{1}^{\prime}(n-1) \\
& =p_{1}(n-1)+\left[p_{1}(n-1)-p_{2}(n-2)\right] \\
& =2 p_{1}(n-1)-p_{2}(n-2) .
\end{aligned}
$$

Reflecting and absorbing boundary conditions may be combined as presented in Eq. (3) to receive reflection coefficients with values $0<r<1$ although the results are far from satisfactory. An alternative method was developed as follows. By substituting value $r=1$ into Eq. (3) yields the following equation for a rigid wall

$$
p_{B, r e f}(n)=2 p_{1}(n-1)-p_{B}(n-2) .
$$

Experimentally it can be seen that the use of this condition results in total reflection with zero absorption. By similarity it is presumed to be possible to combine $p_{B, \text { ref }}$ in Eq. (7) with the first order solution of the Taylor series absorbing
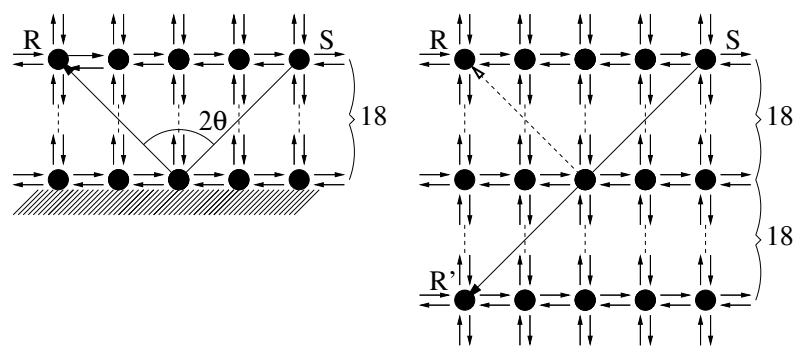

(a)

(b)

Fig. 1. Test setup in the 2-D mesh. The source and the receivers were placed in line (a). The reflection from the boundary was compared to the level of a freely radiated signal measured at location $\mathrm{R}^{\prime}$ in a larger mesh to avoid unwanted reflections (b).

boundary condition $p_{B, a b s}$ in Eq. (6). The combination is weighted by the reflection coefficient $r$ as:

$$
\begin{aligned}
p_{B, r}(n)= & r p_{B, r e f}(n)+(1-r) p_{B, a b s}(n) \\
= & r\left[2 p_{1}(n-1)-p_{B}(n-2)\right]+ \\
& (1-r)\left[2 p_{1}(n-1)-p_{2}(n-2)\right] \\
= & 2 p_{1}(n-1)-r p_{B}(n-2)- \\
& (1-r) p_{2}(n-2) .
\end{aligned}
$$

To further improve the behaviour of the boundary conditions, a second-order linear-phase FIR filter is introduced. The last term in Eq. (7) therefore becomes:

$$
h_{B a} p_{B}(n-2)+\frac{1-h_{B a}}{2}\left[p_{B}(n-1)+p_{B}(n-3)\right],
$$

where $h_{B a}$ is a filter coefficient, $0 \leq h_{B a} \leq 1$, to be optimized numerically. Now Eq. (7) becomes:

$$
\begin{gathered}
p_{B, r e f}(n)=2 p_{1}(n-1)-h_{B a} p_{B}(n-2)- \\
\frac{1-h_{B a}}{2}\left[p_{B}(n-1)+p_{B}(n-3)\right] .
\end{gathered}
$$

The absorbing boundary condition is treated similarly. The Eq. (6) therefore becomes:

$$
\begin{gathered}
p_{B, a b s}(n)_{1}=2 p_{1}(n-1)-h_{B a} p_{2}(n-2)- \\
\frac{1-h_{B a}}{2}\left[p_{1}(n-1)+p_{3}(n-3)\right] .
\end{gathered}
$$

Note that the filtering and hence the absoprtion is now obtained through using consecutive neighbouring nodes, rather than with values of the same node at adjacent time steps as in Eq. (10). This improves the performance of the filter, but can not be done for the reflecting boundary condition directly because the node $p_{B}$, the value of which is filtered, is at the boundary of the mesh, and hence only has one neighbour. 


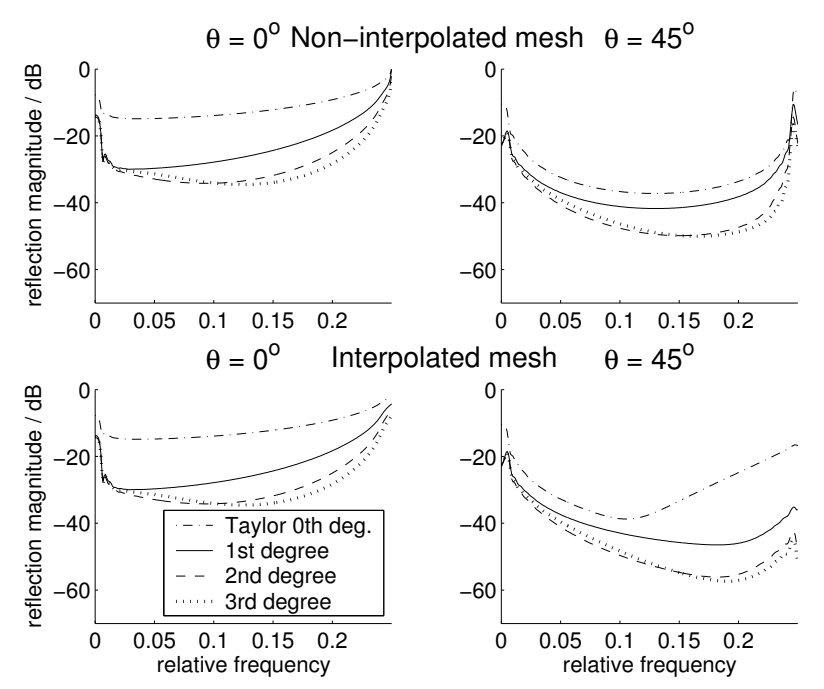

Fig. 2. Reflections from the Taylor-series absorbing boundaries of orders $0 \ldots 3$ in digital waveguide mesh for angles of incidence $\theta=0^{\circ}$ and $\theta=45^{\circ}$.

\section{SIMULATION SETUP AND RESULTS}

Simulations were executed in a 2-D mesh of $600 \times 300$ junctions. The mesh was initialized with a low-pass filtered impulse at the point $(140,18)$. Receivers were located at the same distance from the boundary as indicated in Fig. 1(a). The direct component from source to receiver was removed from the measurement signals. To resolve the achieved absorption the resulting signal level of the reflection was compared to the level of a signal that had passed the same distance without any reflections as illustrated in Fig. 1(b). A Hanning function was used for windowing the last half of the signal to avoid the cutting error in calculation of the spectra.

\subsection{Absorbing boundary condition}

The simulation was run in two dimensions on both interpolated and non-interpolated rectangular meshes. The simulation results for the Taylor series absorbing boundary condition at two incidence angles are presented in Fig. 2. In all the figures the frequency band is shown up to 0.25 the normalized relative frequency since that is the upper frequency limit of the mesh. It can be seen from the results that the first order and the second order solutions reduce the reflected signal power significantly compared to the zero order solution, although the third order solution does not give a corresponding advantage. Thus the second order solution was selected as optimal for future reference. Note that according to Fig. 2 the interpolated mesh does not change the behavior of reflection at angle of incidence $\theta=0^{\circ}$.

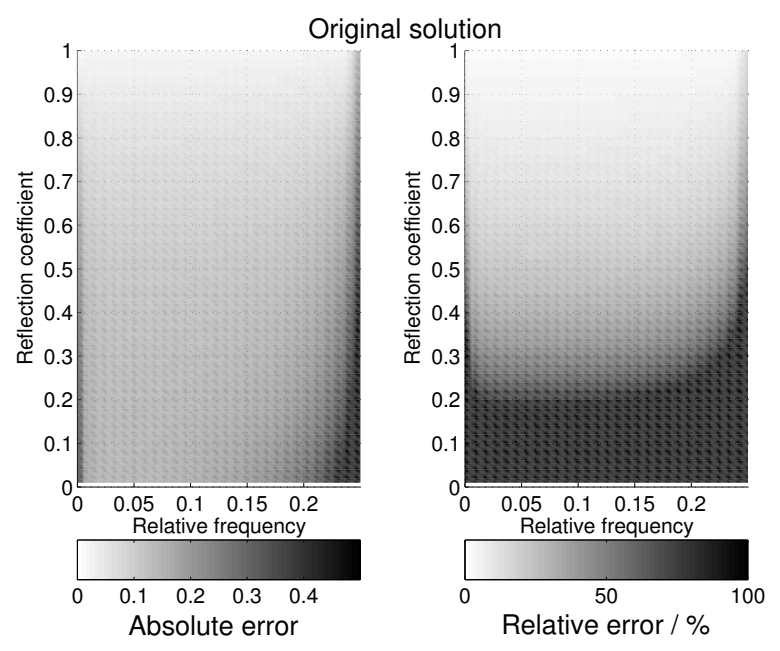

Fig. 3. Maximum absolute and relative error of the received reflection coefficient as a function of reflection coefficient $r$ in the original boundary condition function (3).

\subsection{Arbitrary reflection coefficient}

The boundary conditions for freely adjustable values of $r$ were tested in the same two dimensional mesh as the absorbing boundaries. The maximum error was searched at angles $0^{\circ} \leq \theta \leq 57^{\circ}$. Greater incidence angles were not considered as the directional error caused by the change from two dimensional mesh to one dimensional boundary condition becomes high near the angle $\theta=65^{\circ}$.

As indicated in Fig. 3, results given by Eq. (3) are erroneus especially with small reflection coefficients. Comparing with Fig. 4 shows that the new method given by Eq. (8) is better. The phase delay characteristics of the two methods were also compared. The behaviour of the signal phase is not ideal with either one of the conditions but the new method is as good as the old.

When using the FIR based boundary condition substituting Eqs. (10) and (11) into Eq. (8) the maximum error obtained at the measurement points was minimized by optimization of the filter coefficient $h_{B a}$. It was noted that the filter behaved as expected although the optimal value of $h_{B a}$ was heavily frequency dependent. For stability the values were restricted to $0 \leq h_{B a} \leq 1$. The full range of values were used for $0.06 \leq f \leq 0.1$. At frequencies $f<0.06$ the coefficient was at the minimum and at $f>0.1$ the coefficient was at its maximum allowed value.

To make the best use of the filtered boundary condition, the simulation was divided into two frequency bands about $f=0.09$. To minimize the maximum error the filter coefficient was set to $h_{B a}=0$ for the lower band and $h_{B a}=1$ for the upper band. The level of the relative error was diminished through the use of the filter as shown in Fig. 5. 


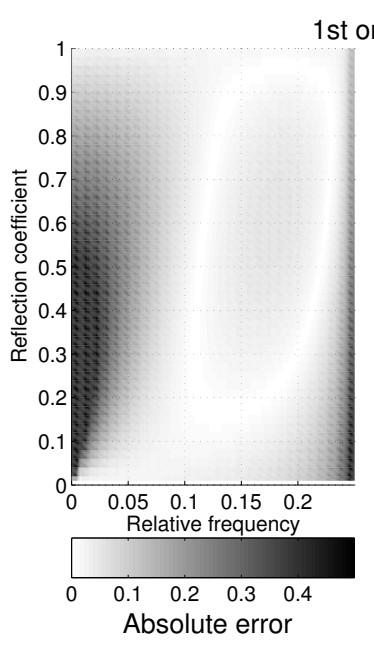

1 st order solution

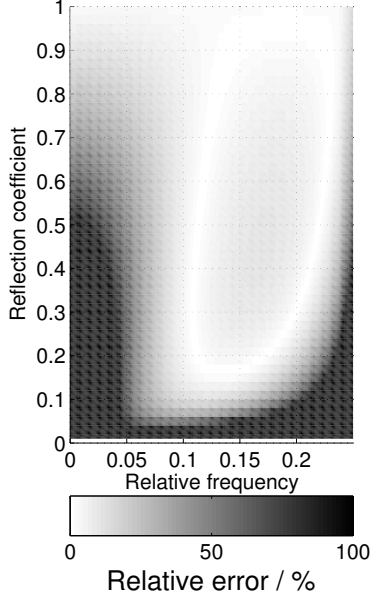

Fig. 4. Maximum absolute and relative error of the received reflection coefficient as a function of reflection coefficient $r$ in the linear combination boundary condition (8).

Frequency bands, for which the maximum relative error is below $15 \%$ at reflection coefficient values $r=0.2, r=$ 0.5 and $r=0.8$ are listed in Table 1. The table shows that compared to the original method, the new boundary conditions allow the use of remarkably smaller values of $r$.

\section{CONCLUSION}

This paper has developed and numerically experimented with the Taylor series absorbing boundary conditions, as introduced previously by Murphy and Mullen. A novel way to create higher order reflecting boundaries with adjustable reflection coefficients has been introduced. The main goal was to calculate a linear combination of reflecting and absorbing boundary conditions. The performance of these borders was improved by use of a second-order linear-phase FIR filter. As the filter is most beneficial at the low frequencies, the calculation was divided into two frequency bands.

In future this research will focus on issues of frequency dependent and directional errors of both magnitude and phase response of the presented boundary conditions.

Table 1. Frequency bands, for which the maximum relative error is below $15 \%$ for the three boundary conditions.

\begin{tabular}{|l|l|l|l|}
\hline method & $r=0.2$ & $r=0.5$ & $r=0.8$ \\
\hline original & - & - & $0-0.24$ \\
1st order & $0.09-0.20$ & $0.09-0.23$ & $0.07-0.24$ \\
filtered & $0.06-0.20$ & $0.05-0.23$ & $0.04-0.24$ \\
\hline
\end{tabular}

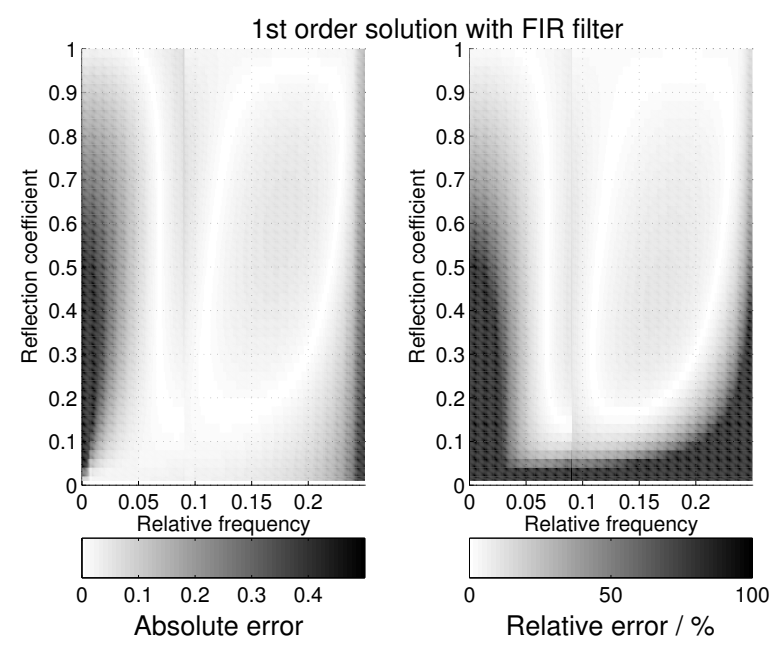

Fig. 5. Maximum absolute and relative error of the received reflection coefficient as a function of reflection coefficient $r$, when filter coefficient is $h_{B a}=0$ at $f \leq 0.09$ and $h_{B a}=1$ when $f>0.09$.

\section{REFERENCES}

[1] S. Van Duyne and J. O. Smith, "The 2-D digital waveguide mesh," in Proc. IEEE Workshop on Applications of Signal Processing to Audio and Acoustics (WASPAA'93), New Paltz, NY, October 1993.

[2] L. Savioja, T. Rinne, and T. Takala, "Simulation of room acoustics with a 3-D finite difference mesh," in Proc. Int. Computer Music Conf. (ICMC'94), Aarhus, Denmark, September 1994, pp. 463-466.

[3] D. T. Murphy and M. J. Beeson, "Modelling spatial sound occlusion and diffraction effects using the digital waveguide mesh," in Proc. AES 24th International Conference, Multichannel Audio, June 2003.

[4] J. O. Smith, "Physical modeling using digital waveguides," Computer Music Journal, vol. 16, no. 4, pp. 74-91, 1992.

[5] L. Savioja and V. Välimäki, "Reducing the dispersion error in the digital waveguide mesh using interpolation and frequency warping techniques," IEEE Trans. Speech and Audio Processing, vol. 8, pp. 184 - 194, March 2000.

[6] D. T. Murphy and J. Mullen, "Digital waveguide mesh modelling of room acoustics: improved anechoic boundaries," in Proc. DAFX-02, Hamburg, Germany, September 2002, pp. $163-168$. 